\title{
Article
}

\section{Stability of Pinelas-Septoicosic functional equation}

\section{Sandra Pinelas ${ }^{1, *}$, Govindan Vediyappan ${ }^{2}$ and Kandhasamy Tamilvanan ${ }^{3}$}

1 Departmento de Cie İĆncias Exatas e Engenharia, Academia Militar, Portugal

2 Sri Vidya Mandir Arts \& Science College, Katteri, Uthangarai, Tamilnadu, 636902, India; govindoviya@gmail.com

3 Department of Mathematics, Government Arts and Science College (For Men), Krishnagiri, Tamil Nadu, 635001

India; tamiltamilk7@gmail.com

* Correspondence: sandra.pinelas@gmail.com

Received: 18 August 2018; Accepted: 31 December 2018; Published: 9 February 2019.

Abstract: In this paper, we find the general solution of a Septoicosic functional equation (11) for all $x, y \in X$ and investigate its general Hyers-Ulam stability in Banach Space using direct and fixed point methods.

Keywords: Banach space, fixed point, Hyers-Ulam stability, Pinelas-Septoicosic functional equation.

MSC: 39B52, 39B72, 39B82.

\section{Introduction}

$\mathbf{T}$ he stability phenomenon presented by Th. M. Rassias is called the generalized Hyers-Ulam stability. In 1994, a generalization of the Rassias theorem was obtained by Gavruta [1] by replacing the unbounded Cauchy difference by a general control function. In [2], Ulam proposed the general Ulam stability problem: "When is it true that by slightly changing the hypotheses of a theorem one can still assert, that the thesis of the theorem remains true or approximately true?" In [3], Hyers gave the first affirmative answer to the question of Ulam for additive functional equations on Banach spaces.

The issue of stability of functional equations has appeared in connection with a question that Ulam [4] posed in 1940. Hyers [3], by using direct method, brilliantly gave a partial answer for the case of the additive Cauchy functional equation for mappings between Banach Spaces. This result was then improved by Aoki [5] and Rassias [6], who weakened the condition for the bound of the norm of the Cauchy difference. Many papers are written in this direction such as [7-14].

We can remember some of the various type of functional equations as follows: One of the most famous functional equations is the additive functional equation

$$
f(x+y)=f(x)+f(y) .
$$

Every solution of the additive functional equation (1) is called an additive function. The theory of additive functional equations is frequently applied to the development of theories of other functional equations. The second famous functional equation

$$
f(x+y)+f(x-y)=2 f(x)+2 f(y)
$$

is said to be quadratic functional equation because the quadratic function $f(x)=a x^{2}$ is a solution of the functional equation (2). Skof's result was further extended by S. Czerwik [15] to the Hyers-Ulam-Rassias stability of the quadratic functional equation (2). Jun and Kim [16] introduced the following cubic functional equation

$$
f(2 x+y)+f(2 x-y)=2 f(x+y)+2 f(x-y)+12 f(x)
$$

and they established the general solution and the generalized Hyers-Ulam stability for the functional equation. The function $f(x)=x^{3}$ satisfies the functional equation (3), which is called a cubic functional equation.

Lee $e t$ al., [17] obtained the general solution of the following quartic functional equation

$$
f(2 x+y)+f(2 x-y)=4 f(x+y)+4 f(x-y)+24 f(x)-6 f(y)
$$


and proved the Hyers-Ulam-Rassias stability of this equation. It is easy to show that the function $f(x)=$ $x^{4}$ satisfies the functional equation (4), which is called a quartic functional equation and every solution of the quartic functional equation is said to be a quartic function. The general solution of Quintic and Sextic functional equations

$$
f(x+3 y)-5 f(x+2 y)+10 f(x+y)-10 f(x)+5 f(x-y)-f(x-2 y)=120 f(y)
$$

and

$$
\begin{aligned}
& f(x+3 y)-6 f(x+2 y)+15 f(x+y)-20 f(x)+15 f(x-y) \\
& -6 f(x-2 y)+f(x-3 y)=720 f(y)
\end{aligned}
$$

was introduced and investigated by Xu et al., [18]. Also, they in [19] introduced and discussed the general solution and generalized Ulam-Hyers stability of Septic and Octic functional equations

$$
\begin{aligned}
& f(x+4 y)-7 f(x+3 y)+21 f(x+2 y)-35 f(x+y)+35 f(x) \\
& -21 f(x-y)+7 f(x-2 y)-f(x-3 y)=5040 f(y)
\end{aligned}
$$

and

$$
\begin{aligned}
& f(x+4 y)-8 f(x+3 y)+28 f(x+2 y)-56 f(x+y)+70 f(x) \\
& -56 f(x-y)+28 f(x-2 y)-8 f(x-3 y)+f(x-4 y)=40320 f(y)
\end{aligned}
$$

in quasi $\beta$-normed spaces, respectively. In [20], they introduced the nonic functional equation as follows:

$$
\begin{aligned}
& f(x+5 y)-9 f(x+4 y)+36 f(x+3 y)-84 f(x+2 y)+126 f(x+y) \\
& -126 f(x)+84 f(x-y)-36 f(x-2 y)+9 f(x-3 y)-f(x-4 y)=9 ! f(y)
\end{aligned}
$$

where $9 !=362880$. Recently, the general solution in vector space and generalized Ulam-Hyers stability of the equation (9) in Banach space and various normed space by using direct and fixed point methods was introduced and investigated in [21] and [22].

Ravi et al., [23] introduced the undecic functional equation as follows:

$$
\begin{aligned}
& f(x+6 y)-11 f(x+5 y)+55 f(x+4 y)-165 f(x+3 y)+330 f(x+2 y)-462 f(x+y) \\
& +462 f(x)-330 f(x-y)+165 f(x-2 y)-55 f(x-3 y)+11 f(x-4 y)-f(x-5 y)=39916800
\end{aligned}
$$

and generalized Ulam-Hyers stability of the above functional equation in quasi- $\beta$ normed spaces using fixed point method. Moreover, they investigate the stabilities of the above functional equation. Murali Ramdoss et al., in [24] carry out the Hyers-Ulam stability of duodecic functional equation in Multi-Banach spaces using fixed point method and also introduced the tredecic functional equation in [25] and investigated and established the generalized Ulam-Hyers stability of this functional equation in matrix normed spaces by using the fixed point method, septndecic functional equation [26] in matrix normed spaces. Nazarlanpoor et al., introduced the Octadecic functional equation in [27] and they proved the stability for multi normed space by using standard fixed point method and also nonadecic functional equation [28] in Multi-Banach spaces. Murali et al., establish the general solution for the viginti duo functional equation in [29] and determined the Hyers-Ulam stability for a the viginti duo functional equation in Multi-Banach spaces by using fixed point technique. Finally, Rassias et al., established the general solution for the Quattuorvigintic functional equation in [30] and also established Hyers-Ulam stability of the Quattuorvigintic functional equation in multi Banach spaces. In this present work, we introduce the following Pinelas-Septoicosic functional equation

$$
\begin{aligned}
& f(x+14 y)-27 f(x+13 y)+351 f(x+12 y)-2925 f(x+11 y)+17550 f(x+10 y)-80730 f(x+9 y) \\
& +296010 f(x+8 y)-888030 f(x+7 y)+2220075 f(x+6 y)-4686825 f(x+5 y)+8436285 f(x+4 y) \\
& -13037895 f(x+3 y)+17383860 f(x+2 y)-20058300 f(x+y)+20058300 f(x)-17383860 f(x-y) \\
& +13037895 f(x-2 y)-8436285 f(x-3 y)+4686825 f(x-4 y)-2220075 f(x-5 y)+888030 f(x-6 y)
\end{aligned}
$$




$$
\begin{aligned}
& -296010 f(x-7 y)+80730 f(x-8 y)-17550 f(x-9 y)+2925 f(x-10 y)-351 f(x-11 y) \\
& +27 f(x-12 y)-f(x-13 y)=27 ! f(y)
\end{aligned}
$$

where $27 !=1.088886945 \times 10^{28}$. We also investigate the generalized Hyers-Ulam stability of this functional equation in Banach Space using direct method and fixed point method. Furthermore, we can refer [31-45].

Theorem 1. (Banach Contraction Principle) Let $(X, d)$ be a complete metric space and consider a mapping $T: X \rightarrow X$ which is strictly contractive mapping, that is

(A1) $d\left(T_{x}, T_{y}\right) \leq L d(x, y)$ for some (Lipschitz constant) $L<1$, then

1. The mapping $T$ has one and only fixed point $x^{*}=T\left(x^{*}\right)$;

2. The fixed point for each given element $x^{*}$ is globally attractive that is

(A2) $\lim _{n \rightarrow \infty} T^{n} x=x^{*}$,for any starting point $x \in X$;

1. One has the following estimation inequalities:

(A3) $d\left(T^{n} x, x^{*}\right) \leq \frac{1}{1-L} d\left(T^{n} x, T^{n+1} x\right)$, for all $n \geq 0, x \in X$.

(A4) $d\left(x, x^{*}\right) \leq \frac{1}{1-L} d\left(x, x^{*}\right), \quad \forall x \in X$.

Theorem 2. (The alternative of fixed point) Suppose that for a complete generalized metric space $(X, d)$ and a strictly contractive mapping $T: X \rightarrow Y$ with Lipschitz constant $L$. Then, for each given element $x \in X$, either

(B1) $d\left(T^{n} x, T^{n+1} x\right)=\infty, \forall n \geq 0$ or

(B2) there exists natural number $n_{0}$ such that:

1. $d\left(T^{n} x, T^{n+1} x\right)<\infty$ for all $n \geq n_{0}$

2. The sequence $\left(T^{n} x\right)$ is convergent to a fixed point $y^{*}$ of $T$

3. $y^{*}$ is the unique fixed point of $T$ in the set $Y=\left\{y \in X: d\left(T^{n} x, y\right)<\infty\right\}$;

4. $d\left(y^{*}, y\right) \leq \frac{1}{1-L} d(y, T y)$ for all $y \in L$.

\section{General Solution for the Functional Equation (11)}

In the following result, a solution of the functional equation (11) is given. For this, let us consider $X$ and $Y$ be real vector spaces. We observe that the general solution has the generalized polynomial form and it specifies the best mapping $f$ as in Theorem 3; see [46].

Theorem 3. If $f: X \rightarrow Y$ be a mapping satisfying (11) for all $x, y \in X$, then $f$ is Pinelas-Septoicosic.

Proof. Letting $(x, y)$ by $(0,0)$ in $(11)$, we finds that $f(0)=0$. Replacing $(x, y)$ by $(0, x)$ in $(11)$, we have

$$
\begin{aligned}
& f(14 x)-27 f(13 x)+351 f(12 x)-2925 f(11 x)+17550 f(10 x)-80730 f(9 x)+296010 f(8 x) \\
& -888030 f(7 x)+2220075 f(6 x)-4686825 f(5 x)+8436285 f(4 x)-13037895 f(3 x)+1738360 f(2 x) \\
& -20058300 f(x)+20058300 f(0)-17383860 f(-x)+13037895 f(-2 x)-8436285 f(-3 x) \\
& +4686825 f(-4 x)-2220075 f(-5 x)+888030 f(-6 x)-296010 f(-7 x)+80730 f(-8 x)-17550 f(-9 x) \\
& +2925 f(-10 x)-351 f(-11 x)+27 f(-12 x)-f(-13 x)=27 ! f(x)
\end{aligned}
$$

for all $x \in X$. Again substituting $(x, y)$ by $(x,-x)$ in (11), we get

$$
\begin{aligned}
& f(-13 x)-27 f(-12 x)+351 f(-11 x)-2925 f(-10 x)+17550 f(-9 x)-80730 f(-8 x)+296010 f(-7 x) \\
& -888030 f(-6 x)+2220075 f(-5 x)-4686825 f(-4 x)+8436285 f(-3 x)-13037895 f(-2 x) \\
& +17383860 f(-x)-20058300 f(0)+20058300 f(x)-17383860 f(2 x)+13037895 f(3 x)-8436285 f(4 x) \\
& +4686825 f(5 x)-2220075 f(6 x)+888030 f(7 x)-296010 f(8 x)+80730 f(9 x)-17550 f(10 x) \\
& +2925 f(11 x)-351 f(12 x)+27 f(13 x)-f(14 x)=27 ! f(-x)
\end{aligned}
$$

for all $x \in X$. Adding (12) and (13), we get

$$
27 ! f(x)+27 ! f(-x)=0
$$


for all $x \in X$. It follows from (13),

$$
f(-x)=-f(x)
$$

for all $x \in X$. Hence $f$ is an odd function. Setting $(x, y)$ by $(0,2 x)$ in (11), we get

$$
\begin{aligned}
& f(28 x)-26 f(26 x)+324 f(24 x)-2574 f(22 x)+14625 f(20 x)-63180 f(18 x)+215280 f(16 x) \\
& -592020 f(14 x)+1332045 f(12 x)-2466750 f(10 x)+3749460 f(8 x)-4601610 f(6 x)+4345965 f(4 x) \\
& -1.088886945 \times 10^{28} f(2 x)=0
\end{aligned}
$$

for all $x \in X$. Substituting $(x, y)$ by $(14 x, x)$ in (11), we get

$$
\begin{aligned}
& f(28 x)-27 f(27 x)+351 f(26 x)-2925 f(25 x)+17550 f(24 x)-80730 f(23 x)+296010 f(22 x) \\
& -888030 f(21 x)+2220075 f(20 x)-4686825 f(19 x)+8436285 f(18 x)-13037895 f(17 x) \\
& +17383860 f(16 x)-20058300 f(15 x)+20058300 f(14 x)-17383860 f(13 x)+13037895 f(12 x) \\
& -8436285 f(11 x)+4686825 f(10 x)-2220075 f(9 x)+888030 f(8 x)-296010 f(7 x)+80730 f(6 x) \\
& -17550 f(5 x)+2925 f(4 x)-351 f(3 x)+27 f(2 x)-f(x)=27 ! f(x)
\end{aligned}
$$

for all $x \in X$. Using (16) and (17), we get

$$
\begin{aligned}
& 27 f(27 x)-377 f(26 x)+2925 f(25 x)-17226 f(24 x)+80730 f(23 x)-298584 f(22 x)+888030 f(21 x) \\
& -2205450 f(20 x)+4686825 f(19 x)-8499465 f(18 x)+13037895 f(17 x)-17168580 f(16 x) \\
& +20058300 f(15 x)-20650320 f(14 x)+17383860 f(13 x)-11705850 f(12 x)+8436285 f(11 x) \\
& -7153575 f(10 x)+2220075 f(9 x)-2861430 f(8 x)+296010 f(7 x)-4682340 f(6 x)+17550 f(5 x) \\
& -4343040 f(4 x)+351 f(3 x)-1.088886945 \times 10^{28} f(2 x)+1.088886945 \times 10^{28} f(x)=0
\end{aligned}
$$

for all $x \in X$. Replacing $(x, y)$ by $(13 x, x)$ in (11) and multiply by 27 , we get

$$
\begin{aligned}
& 27 f(27 x)-729 f(26 x)+9477 f(25 x)-78975 f(24 x)+473850 f(23 x)-2179710 f(22 x)+7992270 f(21 x) \\
& -23976810 f(20 x)+59942025 f(19 x)-126544275 f(18 x)+227779695 f(17 x)-352023165 f(16 x) \\
& +469364220 f(15 x)-541574100 f(14 x)+541574100 f(13 x)-469364220 f(12 x)+352023165 f(11 x) \\
& -227779695 f(10 x)+126544275 f(9 x)-59942025 f(8 x)+23976810 f(7 x)-7992270 f(6 x) \\
& +2179710 f(5 x)-473850 f(4 x)+78975 f(3 x)-9477 f(2 x)-2.939994752 \times 10^{29} f(x)=0
\end{aligned}
$$

for all $x \in X$. From (18) and (19), we obtain

$$
\begin{aligned}
& 352 f(26 x)-6552 f(25 x)+61749 f(24 x)-393120 f(23 x)+1881126 f(22 x)-7104240 f(21 x) \\
& +21771360 f(20 x)-55255200 f(19 x)+118044810 f(18 x)-214741800 f(17 x)+334854585 f(16 x) \\
& -449305920 f(15 x)+520923780 f(14 x)-524190240 f(13 x)+457658370 f(12 x)-343586880 f(11 x) \\
& +220626120 f(10 x)-124324200 f(9 x)+62803455 f(8 x)-23680800 f(7 x)+3309930 f(6 x)-2162160 \\
& \times f(5 x)+4816890 f(4 x)-78624 f(3 x)-1.088886945 \times 10^{28} f(2 x)+3.048883446 \times 10^{29} f(x)=0
\end{aligned}
$$

for all $x \in X$. Switching $(x, y)$ by $(12 x, x)$ in (11) and multiply by 352 in the resulted equation, we get

$$
\begin{aligned}
& 352 f(26 x)-9504 f(25 x)+123552 f(24 x)-1029600 f(23 x)+6177600 f(22 x)-28416960 f(21 x) \\
& +104195520 f(20 x)-312586560 f(19 x)+781466400 f(18 x)-1649762400 f(17 x)+2969572320 f(16 x) \\
& -4589339040 f(15 x)+6119118720 f(14 x)-7060521600 f(13 x)+7060521600 f(12 x)-6119118720 f(11 x) \\
& +4589339040 f(10 x)-2969572320 f(9 x)+1649762400 f(8 x)-781466400 f(7 x)+312586560 f f(6 x) \\
& -104195520 f(5 x)+2841690 f(4 x)-6177600 f(3 x)+1029600 f(2 x)-3.832882047 \times 10^{30} f(x)=0 \quad(21)
\end{aligned}
$$

for all $x \in X$. Using (20) and (21), we have

$2952 f(25 x)-61803 f(24 x)+636480 f(23 x)-4296474 f(22 x)+21312720 f(21 x)-82424160 f(20 x)$ 
$+257331360 f(19 x)-663421590 f(18 x)+1435020600 f(17 x)-2634717735 f(16 x)+4140033120 f(15 x)$

$-5598194940 f(14 x)+6536331360 f(13 x)-6602863230 f(12 x)+5775531840 f(11 x)-4368712920 f(10 x)$ $+2845248120 f(9 x)-1586958945 f(8 x)+757785600 f(7 x)-309276630 f(6 x)+102033360 f(5 x)$

$-23600070 f(4 x)+6098976 f(3 x)-1.088886945 \times 10^{28} f(2 x)+4.137770391 \times 10^{30} f(x)=0$

for all $x \in X$. Letting $(x, y)$ by $(11 x, x)$ in (11) and multiply by 2952 , we ontain

$2952 f(25 x)-79704 f(24 x)+1036152 f(23 x)-8634600 f(22 x)+51807600 f(21 x)-238314960 f(20 x)$ $+873821520 f(19 x)-2621464560 f(18 x)+6553661400 f(17 x)-1.38355074 \times 10^{10} f(16 x)+2.490391332$ $\times 10^{10} f(15 x)-3.848786604 \times 10^{10} f(14 x)+5.131715472 \times 10^{10} f(13 x)-5.92121016 \times 10^{10} f(12 x)$ $+5.92121016 \times 10^{10} f(11 x)-5.131715472 \times 10^{10} f(10 x)+3.848786604 \times 10^{10} f(9 x)-2.490391332$ $\times 10^{10} f(8 x)+1.38355074 \times 10^{10} f(7 x)-6553661400 f(6 x)+2621464560 f(5 x)-873821520 f(4 x)$ $+238314960 f(3 x)-51810552 f(2 x)-3.214394262 \times 10^{31} f(x)=0$

for all $x \in X$. From (22) and (23), we get

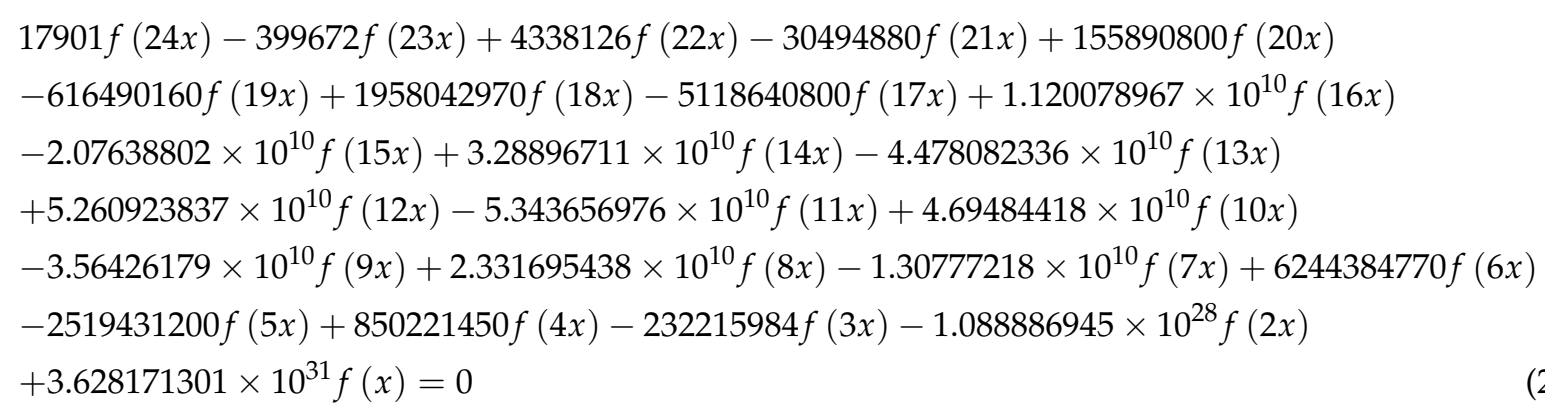

for all $x \in X$. Replacing $(x, y)$ by $(10 x, x)$ in (11) and multiply by 17901 , we get

$17901 f(24 x)-483327 f(23 x)+6283251 f(22 x)-52360425 f(21 x)+314162550 f(20 x)$

$-1445147730 f(19 x)+5298875010 f(18 x)-1.589662503 f(17 x)+3.974156258 \times 10^{10} f(16 x)$

$-8.389885433 \times 10^{10} f(15 x)+1.510179378 \times 10^{11} f(14 x)-2.333913584 \times 10^{11} f(13 x)$

$+3.111884779 \times 10^{11} f(12 x)-3.590636283 \times 10^{11} f(11 x)+3.590636283 \times 10^{11} f(10 x)$

$-3.111884779 \times 10^{11} f(9 x)+2.333913584 \times 10^{11} f(8 x)-1.510179378 \times 10^{11} f(7 x)+8.389885433$

$\times 10^{10} f(6 x)-3.974156258 \times 10^{10} f(5 x)+1.589662503 \times 10^{10} f(4 x)-5298892911 f(3 x)$

$+1445631057 f(2 x)-1.94921652 \times 10^{32} f(x)=0$

for all $x \in X$. It follows from (24) and (25)

$83655 f(23 x)-1945125 f(22 x)+21865545 f(21 x)-158271750 f(20 x)-3340832040 f(18 x)+1.077798423$ $\times 10^{10} f(17 x)-2.854077291 \times 10^{10} f(16 x)+6.313497413 \times 10^{10} f(15 x)-1.181282667 \times 10^{11} f(14 x)$

$+1.88610535 \times 10^{11} f(13 x)-2.585792395 \times 10^{11} f(12 x)+3.056270585 \times 10^{11} f(11 x)$

$-3.121151865 \times 10^{11} f(10 x)+2.755458599 \times 10^{11} f(9 x) 2.10074404 \times 10^{11} f(8 x)+1.37940216 \times 10^{11} f(7 x)$

$-7.765446956 \times 10^{10} f(6 x)+3.722213138 \times 10^{10} f(5 x)-1.504640358 \times 10^{10} f(4 x)$

$+5066641125 f(3 x)-1.088886945 \times 10^{28} f(2 x)+2.31203365 \times 10^{32} f(x)=0$

for all $x \in X$. Substituting $(x, y)$ by $(9 x, x)$ in (11) and multiply by 83655 , we get

$83655 f(23 x)-2258685 f(22 x)+29362905 f(21 x)-244690875 f(20 x)+1468145250 f(19 x)-6753468150$ $f(18 x)+2.476271655 \times 10^{10} f(17 x)-7.428814965 \times 10^{10} f(16 x)+1.857203741 \times 10^{11} f(15 x)$

$-3.920763454 \times 10^{11} f(14 x)+7.057374217 \times 10^{11} f(13 x)-1.090685106 \times 10^{12} f(12 x)+1.454246808 \times 10^{12}$ $f(11 x)-1.677977087 \times 10^{12} f(10 x)+1.677977087 \times 10^{12} f(9 x)-1.454246808 \times 10^{12} f(8 x)$ 
$+1.090685106 \times 10^{12} f(7 x)-7.057374217 \times 10^{11} f(6 x)+3.920763454 \times 10^{11} f(5 x)-1.857204578 \times 10^{11}$ $f(4 x)+7.429040834 \times 10^{10} f(3 x)-2.479207946 \times 10^{10} f(2 x)-9.109083739 \times 10^{32} f(x)=0$

for all $x \in X$. Using (26) and (27), we obtain

$313560 f(22 x)-7497360 f(21 x)+86419125 f(20 x)-639487680 f(19 x)+3412636110 f(18 x)-1.398473232$ $\times 10^{10} f(17 x)+4.574737674 \times 10^{10} f(16 x)-1.225854 \times 10^{11} f(15 x)+2.739480787 \times 10^{11} f(14 x)$

$-5.171268866 \times 10^{11} f(13 x)+8.321058665 \times 10^{11} f(12 x)-1.14861975 \times 10^{12} f(11 x)+1.3658619$

$\times 10^{12} f(10 x)-1.402431227 \times 10^{12} f(9 x)+1.244172404 \times 10^{12} f(8 x)-9.527448902$

$\times 10^{11} f(7 x)+6.280829521 \times 10^{11} f(6 x)-3.54854214 \times 10^{11} f(5 x)+1.706738869 \times 10^{11} f(4 x)$

$-6.921924984 \times 10^{10} f(3 x)-1.088886945 \times 10^{28} f(2 x)+1.142111739 \times 10^{33} f(x)=0$

for all $x \in X$. Setting $(x, y)$ by $(8 x, x)$ in (11) and multiply by 313560 , we have

$313560 f(22 x)-8466120 f(21 x)+110059560 f(20 x)-917163000 f(19 x)+5502978000 f(18 x)$

$-2.53136988 \times 10^{10} f(17 x)+9.28168956 \times 10^{10} f(16 x)-2.784506868 \times 10^{11} f(15 x)$

$+6.96126717 \times 10^{11} f(14 x)-1.469600847 \times 10^{12} f(13 x)+2.645281525 \times 10^{12} f(12 x)-4.088162356$

$\times 10^{12} f(11 x)+5.450883142 \times 10^{12} f(10 x)-6.289480548 \times 10^{12} f(9 x)+6.289480548 \times 10^{12} f(8 x)$

$-5.450883142 \times 10^{12} f(7 x)+4.088162356 \times 10^{12} f(6 x)-2.645281838 \times 10^{12} f(5 x)+1.469609313$

$\times 10^{12} f(4 x)-6.962367766 \times 10^{11} f(3 x)+2.793678498 \times 10^{11} f(2 x)-3.414313905 \times 10^{33} f(x)=0(29)$

for all $x \in X$. From (28) and (29), we get

$968760 f(21 x)-23640435 f(20 x)+277675320 f(19 x)-2090341890 f(18 x)+1.132896648 \times 10^{10} f(17 x)$ $-4.706951886 \times 10^{10} f(16 x)+1.558652868 \times 10^{11} f(15 x)-4.221786383 \times 10^{11} f(14 x)+9.524739604$ $\times 10^{11} f(13 x)-1.813175658 \times 10^{12} f(12 x)+2.939542606 \times 10^{12} f(11 x)-4.085021242 \times 10^{12} f(10 x)$ $+4.887049321 \times 10^{12} f(9 x)-5.045308144 \times 10^{12} f(8 x)+4.498138251 \times 10^{12} f(7 x)-3.46007904 \times 10^{12}$ $f(6 x)+2.290426997 \times 10^{12} f(5 x)-1.298918494 \times 10^{12} f(4 x)+6.267974076 \times 10^{11} f(3 x)$

$-1.088886945 \times 10^{28} f(2 x)+4.556425644 \times 10^{33} f(x)=0$

for all $x \in X$. Replacing $(x, y)$ by $(7 x, x)$ in (11) and multiply by 968760 , we get

$$
\begin{aligned}
& 968760 f(21 x)-26156520 f(20 x)+340034760 f(19 x)-2833623000 f(18 x)+1.7001738 \times 10^{10} f(17 x) \\
& -7.82079948 \times 10^{10} f(16 x)+2.867626476 \times 10^{11} f(15 x)-8.602879428 \times 10^{11} f(14 x) \\
& +2.150719857 \times 10^{12} f(13 x)-4.540408587 \times 10^{12} f(12 x)+8.172735457 \times 10^{12} f(11 x)-1.263059116 \\
& \times 10^{13} f(10 x)+1.684078821 \times 10^{13} f(9 x)-1.943167871 \times 10^{13} f(8 x)+1.943167871 \times 10^{13} f(7 x) \\
& -1.684078724 \times 10^{13} f(6 x)+1.2630565 \times 10^{13} f(5 x)-8.172395422 \times 10^{12} f(4 x)+4.537574964 \\
& \times 10^{12} f(3 x)-2.133718119 \times 10^{12} f(2 x)-1.054870117 \times 10^{34} f(x)=0
\end{aligned}
$$

for all $x \in X$. It follows from (30) and (31)

$2516085 f(20 x)-62359440 f(19 x)+743281110 f(18 x)-5672771520 f(17 x)+3.113847594 \times 10^{10} f(16 x)$ $-1.308973608 \times 10^{11} f(15 x)+4.381093045 \times 10^{11} f(14 x)-1.198245897 \times 10^{12} f(13 x)+2.727232929$

$f(12 x)-5.23319285 \times 10^{12} f(11 x)+8.545569919 \times 10^{12} f(10 x)-1.195373889 \times 10^{13} f(9 x)$

$\times 10^{12}+1.438637056 \times 10^{13} f(8 x)-1.493354046 \times 10^{13} f(7 x)+1.338070784 \times 10^{13} f(6 x)-1.034013801$

$\times 10^{13} f(5 x)+6.873476928 \times 10^{12} f(4 x)-3.910777556 \times 10^{12} f(3 x)-1.088886945 \times 10^{28} f(2 x)$

$+1.510512681 \times 10^{34} f(x)=0$

for all $x \in X$. Substituting $(x, y)$ by $(6 x, x)$ in (11) and multiply by 2516085 , we get

$2516085 f(20 x)-67934295 f(19 x)+883145835 f(18 x)-7359548625 f(17 x)+4.415729175 \times 10^{10} f(16 x)$ 
$-2.031235421 \times 10^{11} f(15 x)+7.447863209 \times 10^{11} f(14 x)-2.031235421 \times 10^{11} f(15 x)+7.447863209$ $\times 10^{11} f(14 x)-2.234358963 \times 10^{12} f(13 x)+5.585897406 \times 10^{12} f(12 x)-1.179245008 \times 10^{13} f(11 x)$ $+2.122641014 \times 10^{13} f(10 x)-3.280445204 \times 10^{13} f(9 x)+4.373926939 \times 10^{13} f(8 x)-5.046838524 \times 10^{13}$ $+5.046831982 \times 10^{13} f(6 x)-4.373838624 \times 10^{13} f(5 x)+3.279709249 \times 10^{13} f(4 x)-2.118225285$ $\times 10^{13} f(3 x)+1.158932654 \times 10^{13} f(2 x)-2.739732109 \times 10^{34} f(x)=0$

for all $x \in X$. Using (32) and (33), we get

$5574855 f(19 x)-139864725 f(18 x)+1686777105 f(17 x)-1.301881581 \times 10^{10} f(16 x)+7.222618125$ $\times 10^{10} f(15 x)-3.066770164 \times 10^{11} f(14 x)+1.036113066 \times 10^{12} f(13 x)-2.858664477$ $\times 10^{12} f(12 x)+6.55925723 \times 10^{12} f(11 x)-1.268084023 \times 10^{13} f(10 x)+2.085071315$ $\times 10^{13} f(9 x)-2.935289882 \times 10^{13} f(8 x)+3.553484478 \times 10^{13} f(7 x)-3.708761198 \times 10^{13} f(6 x)$ $+3.339824824 \times 10^{13} f(5 x)-2.592361556 \times 10^{13} f(4 x)+1.72714753 \times 10^{13} f(3 x)-1.088886945 \times 10^{28}$ $f(2 x)+4.25024479 \times 10^{34} f(x)=0$

for all $x \in X$. Replacing $(x, y)$ by $(5 x, x)$ in (11) and multiply by 5574855 , we have

$5574855 f(19 x)-150521085 f(18 x)+1956774105 f(17 x)-1.630645088 \times 10^{10} f(16 x)+9.783870525$ $\times 10^{10} f(15 x)-4.500580442 \times 10^{11} f(14 x)+1.650212829 \times 10^{12} f(13 x)-4.950638486 \times 10^{12}$ $f(12 x)+1.237659621 \times 10^{13} f(11 x)-2.612836979 \times 10^{13} f(10 x)+4.70310656 \times 10^{13} f(9 x)$ $-7.268436856 \times 10^{13} f(8 x)+9.691234832 \times 10^{13} f(7 x)-1.118201573 \times 10^{14} f(6 x)+1.118058076$ $\times 10^{14} f(5 x)-9.681466014 \times 10^{13} f(4 x)+7.22431609 \times 10^{13} f(3 x)-4.538085279 \times 10^{13} f(2 x)$ $-6.07038683 \times 10^{34} f(x)=0$

for all $x \in X$. From (34) and (35), we get

$$
\begin{aligned}
& 10656360 f(18 x)-269997000 f(17 x)+3287635065 f(16 x)-2.5612524 \times 10^{10} f(15 x)+1.433810278 \\
& \times 10^{11} f(14 x)-6.140997626 \times 10^{11} f(13 x)+2.091974008 \times 10^{12} f(12 x)-5.817338984 \\
& \times 10^{12} f(11 x)+1.344752956 \times 10^{13} f(10 x)-2.618035246 \times 10^{13} f(9 x)+4.333146973 \times 10^{13} \\
& f(8 x)-6.137750354 \times 10^{13} f(7 x)+7.473254529 \times 10^{13} f(6 x)-7.840755936 \times 10^{13} f(5 x) \\
& +7.089104457 \times 10^{13} f(4 x)-5.496284079 \times 10^{13} f(3 x)-1.088886945 \times 10^{28} f(2 x)+1.032063162 \\
& \times 10^{35} f(x)=0
\end{aligned}
$$

for all $x \in X$. Letting $(x, y)$ by $(4 x, x)$ in (11) and multiply by 10656360 , we obtain

$$
\begin{aligned}
& 10656360 f(18 x)-287721720 f(17 x)+3740382360 f(16 x)-3.1169853 \times 10^{10} f(15 x)+1.87019118 \\
& \times 10^{11} f(14 x)-8.602879428 \times 10^{11} f(13 x)+3.154389124 \times 10^{12} f(12 x)-9.463167371 \\
& \times 10^{12} f(11 x)+2.365791843 \times 10^{13} f(10 x)-4.99444838 \times 10^{13} f(9 x)+8.98998023 \times 10^{13} \\
& f(8 x)-1.389327624 \times 10^{14} f(7 x)+1.852175005 \times 10^{14} f(6 x)-2.135614467 \times 10^{14} f(5 x) \\
& +2.128881778 \times 10^{14} f(4 x)-1.820942812 \times 10^{14} f(3 x)+1.294733354 \times 10^{14} f(2 x)-1.160357129 \\
& \times 10^{35} f(x)=0
\end{aligned}
$$

for all $x \in X$. It follows from (36) and (37)

$17724720 f(17 x)-452747295 f(16 x)+5557329000 f(15 x)-4.363809021 \times 10^{10} f(14 x)+2.461881802$ $\times 10^{11} f(13 x)-1.062415115 \times 10^{12} f(12 x)+3.645828387 \times 10^{12} f(11 x)-1.021038887$ $\times 10^{13} f(10 x)+2.376413134 \times 10^{13} f(9 x)-4.656833257 \times 10^{13} f(8 x)+7.755525884 \times 10^{13}$ $f(7 x)-1.104849552 \times 10^{14} f(6 x)+1.351538873 \times 10^{14} f(5 x)-1.419971333 \times 10^{14} f(4 x)$ $+1.271314404 \times 10^{14} f(3 x)-1.088886945 \times 10^{28} f(2 x)+2.192420291 \times 10^{35} f(x)=0$ 
for all $x \in X$. Switching $(x, y)$ by $(3 x, x)$ in (11) and multiply by 17724720 , we get

$$
\begin{aligned}
& 17724720 f(17 x)-478567440 f(16 x)+6221376720 f(15 x)-5.1844806 \times 10^{10} f(14 x)+3.11068836 \\
& \times 10^{11} f(13 x)-1.430916646 \times 10^{12} f(12 x)+5.246694367 \times 10^{12} f(11 x)-1.574006538 \\
& \times 10^{13} f(10 x)+3.934972919 \times 10^{13} f(9 x)-8.306643944 \times 10^{13} f(8 x)+1.494789447 \\
& \times 10^{14} f(7 x)-2.307819694 \times 10^{14} f(6 x)+3.066931344 \times 10^{14} f(5 x)-3.502810568 \\
& \times 10^{14} f(4 x)+3.397876681 \times 10^{14} f(3 x)-2.687738433 \times 10^{14} f(2 x)-1.930021621 \times 10^{35} f(x)=0(39)
\end{aligned}
$$

for all $x \in X$. From (38) and (39), we get

$$
\begin{aligned}
& 25820145 f(16 x)-664047720 f(15 x)+8206715790 f(14 x)-6.488065584 \times 10^{10} f(13 x)+3.685015304 \\
& \times 10^{11} f(12 x)-1.600865981 \times 10^{12} f(11 x)+5.52967651 \times 10^{12} f(10 x)-1.558559785 \\
& \times 10^{13} f(9 x)+3.649810687 \times 10^{13} f(8 x)-7.192368582 \times 10^{13} f(7 x)+1.202970142 \times 10^{14} \\
& f(6 x)-1.715392471 \times 10^{14} f(5 x)+2.082839235 \times 10^{14} f(4 x)-2.126562276 \times 10^{14} f(3 x) \\
& -1.088886945 \times 10^{28} f(2 x)+4.122441912 \times 10^{35} f(x)=0
\end{aligned}
$$

for all $x \in X$. Letting $(x, y)$ by $(2 x, x)$ in (11) and multiply by 25820145 , we get

$$
\begin{aligned}
& 25820145 f(16 x)-697143915 f(15 x)+9062870895 f(14 x)-7.552392413 \times 10^{10} f(13 x)+4.531435448 \\
& \times 10^{11} f(12 x)-2.084434486 \times 10^{12} f(11 x)+7.642323978 \times 10^{12} f(10 x)-2.292000049 \times \\
& 10^{13} f(9 x)+5.724713449 \times 10^{13} f(8 x)-1.205613575 \times 10^{14} f(7 x)+2.157416417 \times 10^{14} f(6 x) \\
& -3.289973183 \times 10^{14} f(5 x)+4.259247225 \times 10^{14} f(4 x)-4.60585556 \times 10^{14} f(3 x)+3.968937134 \\
& \times 10^{14} f(2 x)-2.811521881 \times 10^{35} f(x)=0
\end{aligned}
$$

for all $x \in X$. It follows from (40) and (41), we achieve

$$
\begin{aligned}
& 33096195 f(15 x)-856155105 f(14 x)+1.064326829 \times 10^{10} f(13 x)-8.464201434 \times 10^{10} f(12 x) \\
& +4.835685051 \times 10^{11} f(11 x)-2.112647468 \times 10^{12} f(10 x)+7.334402643 \times 10^{12} f(9 x) \\
& -2.074902762 \times 10^{13} f(8 x)+4.863767173 \times 10^{13} f(7 x)-9.544462743 \times 10^{13} f(6 x)+1.574580712 \\
& \times 10^{14} f(5 x)-2.17640799 \times 10^{14} f(4 x)+2.479293284 \times 10^{14} f(3 x)-1.088886945 \\
& \times 10^{28} f(2 x)+6.933963793 \times 10^{35} f(x)=0
\end{aligned}
$$

for all $x \in X$. Substituting $(x, y)$ by $(x, x)$ in (11) and multiply by 33096195, we get

$$
\begin{aligned}
& 33096195 f(15 x)-893597265 f(14 x)+1.161676445 \times 10^{10} f(13 x)-9.677327418 \times 10^{10} f(12 x) \\
& +5.79944625 \times 10^{11} f(11 x)-2.660239058 \times 10^{12} f(10 x)+9.699998312 \times 10^{12} f(9 x)-2.880957582 \\
& \times 10^{13} f(8 x)+7.080417929 \times 10^{13} f(7 x)-1.453192694 \times 10^{14} f(6 x)+2.498185194 \\
& \times 10^{14} f(5 x)-3.580286802 \times 10^{14} f(4 x)+4.202235463 \times 10^{14} f(3 x)-3.846444747 \\
& \times 10^{14} f(2 x)-3.603801467 \times 10^{35} f(x)=0
\end{aligned}
$$

for all $x \in X$. Using (42) and (43), we obtain

$$
\begin{aligned}
& 37442160 f(14 x)-973496160 f(13 x)+1.213125984 \times 10^{10} f(12 x)-9.637611984 \times 10^{10} f(11 x) \\
& +5.4759159 \times 10^{11} f(10 x)-2.365595669 \times 10^{12} f(9 x)+8.060548205 \times 10^{12} f(8 x)-2.216650756 \\
& \times 10^{13} f(7 x)+4.987464202 \times 10^{13} f(6 x)-9.236044818 \times 10^{13} f(5 x)+1.403878812 \\
& \times 10^{14} f(4 x)-1.722942179 \times 10^{14} f(3 x)-1.088886945 \times 10^{28} f(2 x)+1.053776526 \times 10^{36} f(x)=0(44)
\end{aligned}
$$

for all $x \in X$. Replacing $(x, y)$ by $(0, x)$ in (11) and multiply by 37442160 , we have

$37442160 f(14 x)-973496160 f(13 x)+1.213125984 \times 10^{10} f(12 x)-9.637611984 \times 10^{10} f(11 x)$ $+5.4759159 \times 10^{11} f(10 x)-2.365595669 \times 10^{12} f(9 x)+8.060548205 \times 10^{12} f(8 x)-2.216650756$ 


$$
\begin{aligned}
& \times 10^{13} f(7 x)+4.987464202 \times 10^{13} f(6 x)-9.236044818 \times 10^{13} f(5 x)+1.403878812 \times 10^{14} f(4 x) \\
& -1.722942179 \times 10^{14} f(3 x)+1.627223169 \times 10^{14} f(2 x)-4.077027922 \times 10^{35} f(x)=0
\end{aligned}
$$

for all $x \in X$. From (44) and (45), we get $f(2 x)=2^{27} f(x)$.

\section{Stability result of the functional equation (11): Direct Method}

In this section, we investigate the stability result for the new type of functional equation (11) in Banach space using direct method.

Let us consider $X$ be a normed space and $Y$ be a Banach Space. Define a mapping $D f_{27}: X \rightarrow Y$ by

$$
\begin{aligned}
D f_{27}(x, y)= & f(x+14 y)-27 f(x+13 y)+351 f(x+12 y)-2925 f(x+11 y)+17550 f(x+10 y) \\
& -80730 f(x+9 y)+296010 f(x+8 y)-888030 f(x+7 y)+2220075 f(x+6 y) \\
& -4686825 f(x+5 y)+8436285 f(x+4 y)-13037895 f(x+3 y)+17383860 f(x+2 y) \\
& -20058300 f(x+y)+20058300 f(x)-17383860 f(x-y)+13037895 f(x-2 y) \\
& -8436285 f(x-3 y)+4686825 f(x-4 y)-2220075 f(x-5 y)+888030 f(x-6 y) \\
& -296010 f(x-7 y)+80730 f(x-8 y)-17550 f(x-9 y)+2925 f(x-10 y) \\
& -351 f(x-11 y)+27 f(x-12 y)-f(x-13 y)-27 ! f(y)
\end{aligned}
$$

where $27 !=1.088886945 \times 10^{28}$ for all $x, y \in X$.

Theorem 4. Let $j= \pm 1$ and $\alpha: X^{2} \rightarrow[0, \infty)$ be a function such that $\sum_{i=0}^{\infty} \frac{\alpha\left(2^{i j} x, 2^{i j} y\right)}{2^{27 j}}$, converges in $R$ and $\lim _{i \rightarrow \infty} \frac{\alpha\left(2^{i j} x, 2^{i j} y\right)}{2^{27 i j}}=0$ for all $x, y \in X$. Let $D f_{27}: X \rightarrow Y$ be a mapping satisfying the inequality

$$
\left\|D f_{27}(x, y)\right\| \leq \alpha(x, y)
$$

for all $x, y \in X$. Then, there exists a unique mapping $G: X \rightarrow Y$ which satisfies (11) and

$$
\|f(x)-G(x)\| \leq \frac{1}{2^{27}} \sum_{i=\frac{1-j}{2}}^{\infty} \frac{\alpha\left(2^{i j} x, 2^{i j} y\right)}{2^{27 i j}}
$$

where $\alpha\left(2^{i j} x, 2^{i j} x\right)$ and $G(x)$ are defined by

$$
\begin{aligned}
& \alpha\left(2^{i j} x, 2^{i j} x\right)=\frac{1}{1.088886945 \times 10^{28}}\left[\alpha\left(0,2.2^{i j} x\right)+\alpha\left(14.2^{i j} x, 2^{i j} x\right)+27 \alpha\left(13.2^{i j} x, 2^{i j} x\right)\right. \\
& +352 \alpha\left(12.2^{i j} x, 2^{i j} x\right)+2952 \alpha\left(11.2^{i j} x, 2^{i j} x\right)+17901 \alpha\left(10.2^{i j} x, 2^{i j} x\right)+83655 \alpha\left(9.2^{i j} x, 2^{i j} x\right) \\
& +313560 \alpha\left(8.2^{i j} x, 2^{i j} x\right)+968760 \alpha\left(7.2^{i j} x, 2^{i j} x\right)+2516085 \alpha\left(6.2^{i j} x, 2^{i j} x\right)+5574855 \alpha\left(5.2^{i j} x, 2^{i j} x\right) \\
& +10656360 \alpha\left(4.2^{i j} x, 2^{i j} x\right)+17724720 \alpha\left(3.2^{i j} x, 2^{i j} x\right)+25820145 \times \alpha\left(2.2^{i j} x, 2^{i j} x\right)+33096195 \alpha\left(2^{i j} x, 2^{i j} x\right) \\
& +37442160 \alpha\left(0,2^{i j} x\right)
\end{aligned}
$$

and

$$
G(x)=\lim _{i \rightarrow \infty} \frac{f\left(2^{i j} x\right)}{2^{27 i j}}
$$

for all $x \in X$, respectively.

Proof. Replacing $(x, y)$ by $(0,2 x)$ in $(46)$, we obtain

$$
\begin{aligned}
& \| f(28 x)-27 f(26 x)+351 f(24 x)-2925 f(22 x)+17550 f(20 x)-80730 f(18 x)+296010 f(16 x) \\
& -888030 f(14 x)+2220075 f(12 x)-4686825 f(10 x)+8436285 f(8 x)-13037895 f(6 x)+1738360 f(4 x) \\
& -20058300 f(2 x)+20058300 f(0)-17383860 f(-2 x)+13037895 f(-4 x)-8436285 f(-6 x) \\
& +4686825 f(-8 x)-2220075 f(-10 x)+888030 f(-12 x)-296010 f(-14 x) \| \leq \alpha(0,2 x)
\end{aligned}
$$


for all $x \in X$. Setting $(x, y)$ by $(14 x, x)$ in $(46)$, we have

$$
\begin{aligned}
& \| f(28 x)-27 f(27 x)+351 f(26 x)-2925 f(25 x)+17550 f(24 x)-80730 f(23 x) \\
& +296010 f(22 x)-888030 f(21 x)+2220075 f(20 x)-4686825 f(19 x)+8436285 f(18 x)-13037895 f(17 x) \\
& +17383860 f(16 x)-20058300 f(15 x)+20058300 f(14 x)-17383860 f(13 x)+13037895 f(12 x) \\
& -8436285 f(11 x)+4686825 f(10 x)-2220075 f(9 x)+888030 f(8 x)-296010 f(7 x)+80730 f(6 x) \\
& -17550 f(5 x)+2925 f(4 x)-351 f(3 x)+27 f(2 x)-f(x)-27 ! f(x) \| \leq \alpha(14 x, x)
\end{aligned}
$$

for all $x \in X$. Using (50) and (51), we get

$$
\begin{aligned}
& \| 27 f(27 x)-377 f(26 x)+2925 f(25 x)-17226 f(24 x)+80730 f(23 x)-298584 f(22 x) \\
& +888030 f(21 x)-2205450 f(20 x)+4686825 f(19 x)-8499465 f(18 x)+13037895 f(17 x)-17168580 \\
& f(16 x)+20058300 f(15 x)-20650320 f(14 x)+17383860 f(13 x)-11705850 f(12 x)+8436285 f(11 x) \\
& -7153575 f(10 x)+2220075 f(9 x)-2861430 f(8 x)+296010 f(7 x)-4682340 f(6 x)+17550 f(5 x) \\
& -4343040 f(4 x)+351 f(3 x)-1.088886945 \times 10^{28} f(2 x)+1.088886945 \times 10^{28} f(x) \| \\
& \leq \alpha(0,2 x)+\alpha(14 x, x)
\end{aligned}
$$

for all $x \in X$. Switching $(x, y)$ by $(13 x, x)$ in (46) and multiply by 27 , we obtain

$$
\begin{aligned}
& \| 27 f(27 x)-729 f(26 x)+9477 f(25 x)-78975 f(24 x)+473850 f(23 x)-2179710 f(22 x) \\
& +7992270 f(21 x)-23976810 f(20 x)+59942025 f(19 x)-126544275 f(18 x)+227779695 f(17 x) \\
& -352023165 f(16 x)+469364220 f(15 x)-541574100 f(14 x)+541574100 f(13 x)-469364220 f(12 x) \\
& -7992270 f(6 x)+2179710 f(5 x)-473850 f(4 x)+78975 f(3 x)-9477 f(2 x)-2.939994752 \\
& \times 10^{29} f(x) \| \leq 27 \alpha(13 x, x)
\end{aligned}
$$

for all $x \in X$. It follows from (52) and (53)

$$
\begin{aligned}
& \| 352 f(26 x)-6552 f(25 x)+61749 f(24 x)-393120 f(23 x)+1881126 f(22 x) \\
& -7104240 f(21 x)+21771360 f(20 x)-55255200 f(19 x)+118044810 f(18 x)-214741800 f(17 x) \\
& +334854585 f(16 x)-449305920 f(15 x)+520923780 f(14 x)-524190240 f(13 x)+457658370 f(12 x) \\
& -343586880 f(11 x)+220626120 f(10 x)-124324200 f(9 x)+62803455 f(8 x)-23680800 f(7 x) \\
& +3309930 f(6 x)-2162160 f(5 x)+4816890 f(4 x)-78624 f(3 x)-1.088886945 \times 10^{28} f(2 x) \\
& +3.048883446 \times 10^{29} f(x) \| \leq \alpha(0,2 x)+\alpha(14 x, x)+27 \alpha(13 x, x)
\end{aligned}
$$

for all $x \in X$. It follows from the Theorem 3 , we get

$$
\begin{aligned}
& \left\|f(2 x)-2^{27} f(x)\right\| \leq \frac{1}{1.088886945 \times 10^{28}}[\alpha(0,2 x)+\alpha(14 x, x)+27 \alpha(13 x, x)+352 \alpha(12 x, x) \\
& +2952 \alpha(11 x, x)+17901 \alpha(10 x, x)+83655 \alpha(9 x, x)+313560 \alpha(8 x, x)+968760 \alpha(7 x, x)+2516085 \alpha(6 x, x) \\
& +5574855 \alpha(5 x, x)+10656360 \alpha(4 x, x)+17724720 \alpha(3 x, x)+25820145 \alpha(2 x, x)+33096195 \alpha(x, x) \\
& +37442160 \alpha(0, x)]
\end{aligned}
$$

for all $x \in X$. Define

$$
\begin{aligned}
& \alpha(x, x)=\frac{1}{1.088886945 \times 10^{28}}[\alpha(0,2 x)+\alpha(14 x, x)+27 \alpha(13 x, x)+352 \alpha(12 x, x)+2952 \alpha(11 x, x) \\
& +17901 \alpha(10 x, x)+83655 \alpha(9 x, x)+313560 \alpha(8 x, x)+968760 \alpha(7 x, x)+2516085 \alpha(6 x, x) \\
& +5574855 \alpha(5 x, x)+10656360 \alpha(4 x, x)+17724720 \alpha(3 x, x)+25820145 \alpha(2 x, x) \\
& +33096195 \alpha(x, x)+37442160 \alpha(0, x)]
\end{aligned}
$$

for all $x \in X$. From (56), we obtain

$$
\left\|f(2 x)-2^{27} f(x)\right\| \leq \alpha(x, x)
$$


for all $x \in X$. It follows from (57), we get

$$
\left\|\frac{f(2 x)}{2^{27}}-f(x)\right\| \leq \frac{\alpha(x, x)}{2^{27}}
$$

for all $x \in X$. Now, replacing $x$ by $2 x$ and dividing $2^{27}$ in (58), we get

$$
\left\|\frac{f\left(2^{2} x\right)}{2^{54}}-\frac{f(2 x)}{2^{27}}\right\| \leq \frac{\alpha(2 x, 2 x)}{2^{54}}
$$

for all $x \in X$. From (58) and (59), we obtain

$$
\left\|\frac{f\left(2^{2} x\right)}{2^{54}}-f(x)\right\| \leq \frac{1}{2^{27}}\left[\alpha(x, x)+\frac{\alpha(2 x, 2 x)}{2^{27}}\right]
$$

for all $x \in X$. Generalizing for any positive integer $a$, we get

$$
\left\|\frac{f\left(2^{a} x\right)}{2^{a(27)}}-f(x)\right\| \leq \frac{1}{2^{27}} \sum_{i=0}^{a-1} \frac{\alpha\left(2^{i} x, 2^{i} x\right)}{2^{i(27)}}
$$

for all $x \in X$. To prove the convergence of the sequence $\left\{\frac{f\left(2^{a} x\right)}{2^{a(27)}}\right\}$, we replace $x$ by $2^{i} x$ in (61) and divide the resultant by $2^{i(27)}$, for any $a, i>0$, we get

$$
\begin{aligned}
\left\|\frac{f\left(2^{a+i} x\right)}{2^{a+i(27)}}-\frac{f\left(2^{i} x\right)}{2^{i(27)}}\right\| & =\frac{1}{2^{27 i}}\left\|\frac{f\left(2^{a} \cdot 2^{i} x\right)}{2^{a(27)}}-f\left(2^{i} x\right)\right\| \\
& \leq \frac{1}{2^{i(27)}} \frac{1}{2^{27}} \sum_{c=0}^{a-1} \frac{\alpha\left(2^{c} \cdot 2^{i} x, 2^{c} \cdot 2^{i} x\right)}{2^{c(27)}} \\
& \leq \frac{1}{2^{27}} \sum_{c=0}^{\infty} \frac{\alpha\left(2^{c+i} x, 2^{c+i} x\right)}{2^{27(c+i)}} \\
& \rightarrow 0 \text { as } i \rightarrow \infty
\end{aligned}
$$

for all $x \in X$. Thus, it follows that the sequence $\left\{\frac{f\left(2^{a} x\right)}{2^{a(27)}}\right\}$ is Cauchy in $Y$ and so it converges. Therefore, the mapping $G: X \rightarrow Y$ is defined by

$$
G(x)=\lim _{a \rightarrow \infty} \frac{f\left(2^{a} x\right)}{2^{a(27)}}
$$

is well defined for all $x \in X$. In order to show that $G$ satisfies (11) by interchanging $(x, y)$ by $\left(2^{a} x, 2^{a} y\right)$ in $(46)$ and then dividing by $2^{a(27)}$, we get

$$
\begin{aligned}
\|G(x, y)\| & =\lim _{a \rightarrow \infty} \frac{1}{2^{a(27)}}\left\|D f_{27}\left(2^{a} x, 2^{a} y\right)\right\| \\
& \leq \lim _{a \rightarrow \infty} \frac{1}{2^{a(27)}} \alpha\left(2^{a} x, 2^{a} y\right)
\end{aligned}
$$

for all $x, y \in X$. So, the mapping $G$ is Pinelas-Septoicosic.

Taking the limit as $a$ approaches to infinity in (61), we find that the mapping $G$ is a Pinelas-Septoicosic mapping satisfying the inequality (47) near the approximate mapping $f: X \rightarrow Y$ of equation (11). Hence G satisfies (11), for all $x, y \in X$. To prove that $G$ is unique, we assume now that there is $U$ as another Pinelas-Septoicosic mapping satisfying (11) and the inequality (47). Thus

$$
\begin{aligned}
\|G(x)-U(x)\| & =\frac{1}{2^{a(27)}}\left\|G\left(2^{a} x\right)-U\left(2^{a} x\right)\right\| \\
& \leq \frac{1}{2^{a(27)}}\left\{\left\|G\left(2^{a} x\right)-f\left(2^{a} x\right)\right\|+\left\|f\left(2^{a} x\right)-U\left(2^{a} x\right)\right\|\right\} \leq \frac{1}{2^{27}} \sum_{i=0}^{\infty} \frac{\alpha\left(2^{c+a} x, 2^{c+a} x\right)}{2^{27(c+a)}}
\end{aligned}
$$


for all $x \in X$. Therefore, as $a \rightarrow \infty$ in the above inequality, we conclude that $G(x)=U(x)$, for all $x \in X$. Now, Replacing $x$ by $\frac{x}{2}$ in 57 , we have

$$
\left\|f(x)-2^{27} f\left(\frac{x}{2}\right)\right\| \leq \alpha\left(\frac{x}{2}, \frac{x}{2}\right)
$$

for all $x \in X$. The rest of the proof is similar to that of case $j=1$. Thus, for $j=-1$ the assertion holds as well. This completes the proof.

The following corollaries are immediate consequence of Theorem 4 concerning the stability of (11).

Remark 1. Let $D f_{27}: X \rightarrow Y$ be a mapping. If there exist real numbers $\psi$ and $\lambda$ such that

$$
\left\|D f_{27}(x, y)\right\| \leq \begin{cases}\psi, & \\ \psi\left\{\|x\|^{\lambda}+\|y\|^{\lambda}\right\}, & \lambda \neq 27 \\ \psi\|x\|^{\lambda}\|y\|^{\lambda} & \lambda \neq \frac{27}{2} \\ \psi\left\{\|x\|^{\lambda}\|y\|^{\lambda}+\left\{\|x\|^{2 \lambda}+\|y\|^{2 \lambda}\right\}\right\}, & \lambda \neq \frac{27}{2}\end{cases}
$$

for all $x, y \in X$. Then there exists a unique Pinelas-Septoicosic mapping $G: X \rightarrow Y$ such that

$$
\|f(x)-G(x)\| \leq\left\{\begin{array}{c}
\frac{\psi_{C}}{\left|2^{27}-1\right|}, \\
\frac{\psi_{S}\|x\|^{\lambda}}{\mid 2^{27}-2^{\lambda}} \\
\frac{\psi_{P}\|x\|^{2 \lambda}}{\left|2^{27}-2^{2 \lambda}\right|} \\
\frac{\psi_{S}\|x\|^{2 \lambda}}{\left|2^{27}-2^{2 \lambda}\right|}
\end{array}\right.
$$

where,

$$
\begin{aligned}
& l \psi_{C}=\frac{134217729 \psi}{1.088886945 \times 10^{28}} \\
& l \psi_{S}=\frac{\psi}{1.088886945 \times 10^{28}}\left[14^{\lambda}+27.13^{\lambda}+352.12^{\lambda}+2952.11^{\lambda}+17901.10^{\lambda}\right. \\
& +83655.9^{\lambda}+313560.8^{\lambda}+968760.7^{\lambda}+2516085.6^{\lambda}+5574855.5^{\lambda} \\
& \left.+10656360.4^{\lambda}+17724720.3^{\lambda}+25820146.2^{\lambda}+167313923\right] \text {, } \\
& l \psi_{P}=\frac{\psi}{1.088886945 \times 10^{28}}\left[14^{\lambda}+27.13^{\lambda}+352.12^{\lambda}+2952.11^{\lambda}+17901.10^{\lambda}\right. \\
& +83655.9^{\lambda}+313560.8^{\lambda}+968760.7^{\lambda}+2516085.6^{\lambda}+5574855.5^{\lambda} \\
& \left.+10656360.4^{\lambda}+17724720.3^{\lambda}+25820146.2^{\lambda}+33096195\right] \text {, } \\
& l \psi_{S P}=\frac{\psi}{1.088886945 \times 10^{28}}\left\{\left[14^{2 \lambda}+27.13^{2 \lambda}+352.12^{2 \lambda}+2952.11^{2 \lambda}\right.\right. \\
& +17901.10^{2 \lambda}+83655.9^{2 \lambda}+313560.8^{2 \lambda}+968760.7^{2 \lambda}+2516085.6^{2 \lambda} \\
& \left.+5574855.5^{2 \lambda}+10656360.4^{2 \lambda}+17724720.3^{2 \lambda}+25820146.2^{2 \lambda}+167313923\right] \\
& +\left[14^{\lambda}+27.13^{\lambda}+352.12^{\lambda}+2952.11^{\lambda}+17901.10^{\lambda}+83655.9^{\lambda}+313560.8^{\lambda}\right. \\
& +968760.7^{\lambda}+2516085.6^{\lambda}+5574855.5^{\lambda}+10656360.4^{\lambda}+17724720.3^{\lambda} \\
& \left.\left.+25820146 \cdot 2^{\lambda}+33096195\right]\right\}
\end{aligned}
$$

for all $x \in X$. 


\section{Stability result of the functional equation (11): Fixed Point Method}

In this section, we establish the stability result for the new type of functional equation (11) in banach space using fixed point method.

Theorem 5. Let $D f_{27}: X \rightarrow Y$ be a mapping for which there exists a function $\gamma: X^{2} \rightarrow[0, \infty)$ with the condition

$$
\lim _{n \rightarrow \infty} \frac{1}{\eta_{i}^{27 n}} \gamma\left(\eta_{i}^{n} x, \eta_{i}^{n} y\right)=0
$$

where $\eta_{i}=\left\{\begin{array}{ll}2 & \text { if } i=0 \\ \frac{1}{2} & \text { if } i=1\end{array}\right.$, such that the functional inequality

$$
\left\|D f_{27}(x, y)\right\| \leq \gamma(x, y)
$$

holds for all $x, y \in X$. Assume that there exists $L=L(i)$ such that the mapping

$$
D(x, x)=\alpha\left(\frac{x}{2}, \frac{x}{2}\right)
$$

where $\alpha(x, x)$ is defined in (48) with the property

$$
\frac{1}{\eta_{i}^{27}} D\left(\eta_{i} x, \eta_{i} x\right)=L D(x, x)
$$

for all $x \in X$. Then, there exists a unique Pinelas-Septoicosic mapping $G: X \rightarrow Y$ satisfying the functional equation (11) and

$$
\|f(x)-G(x)\| \leq\left(\frac{L^{1-i}}{1-L}\right) D(x, x)
$$

for all $x \in X$.

Proof. Consider the set $Z=\{h \mid h: X \rightarrow Y, h(0)=0\}$ and introduce the generalized metric $d: Z \times Z \rightarrow[0, \infty]$ as follows:

$$
d(g, h)=\inf \{\omega \in(0, \infty):\|g(x)-h(x)\| \leq \omega D(x, x), x \in X\}
$$

It is easy to see that $(Z, d)$ is complete with respect to the defined metric. Let us define the linear mapping $J: Z \rightarrow Z$ by

$$
J h(x)=\frac{1}{\eta_{i}^{27}} h\left(\eta_{i} x\right),
$$

for all $x \in X$. For given $h, g \in Z$, let $\omega \in[0, \infty)$ be an arbitrary constant with $d(g, h) \leq \omega$, that is

$$
\|h(x)-g(x)\| \leq \omega D(x, x)
$$

for all $x \in X$. So, we have

$$
\|J h(x)-J g(x)\|_{X}=\left\|\frac{1}{\eta_{i}^{27}} h\left(\eta_{i} x\right)-\frac{1}{\eta_{i}^{27}} g\left(\eta_{i} x\right)\right\| \leq \frac{\omega}{\eta_{i}^{27}} D\left(\eta_{i} x, \eta_{i} x\right) \leq L \omega D(x, x)
$$

for all $x \in X$, that is $d(J g, J h) \leq L d(g, h)$ for all $g, h \in Z$. This implies that $J$ is a strictly contractive mapping on $Z$ with Lipschitz constant $L$. From (57), (64) and (66) for the case $i=0$, we have

$$
\left\|f(2 x)-2^{27} f(x)\right\| \leq \alpha(x, x)
$$

for all $x \in X$ and

$$
\left\|\frac{f(2 x)}{2^{27}}-f(x)\right\| \leq \frac{1}{2^{27}} \alpha(x, x)
$$

for all $x \in X$. So, we obtain

$$
\|J f(x)-f(x)\| \leq L D(x, x)
$$


for all $x \in X$. Hence,

$$
d(J f, f) \leq L^{1-0}
$$

for all $x \in X$. Replacing $x$ by $\frac{x}{2}$ in (64) and (67) for the case $i=1$, we find

$$
\left\|f(x)-2^{27} f\left(\frac{x}{2}\right)\right\| \leq \alpha\left(\frac{x}{2}, \frac{x}{2}\right)
$$

for all $x \in X$. Then,

$$
\|f(x)-J f(x)\| \leq D(x, x)
$$

for all $x \in X$ and

$$
\|f(x)-J f(x)\| \leq L^{1-1} D(x, x)
$$

for all $x \in X$. Thus, we obtain

$$
d(J f, f) \leq L^{1-1}
$$

for all $f \in Z$. Therefore, from (67) and (68), we arrive

$$
d(J f, f) \leq L^{1-i}
$$

for all $f \in Z$, where $i=0,1$. Hence, the property (B1) holds. It follows from property (B2) that there exists a fixed point $G$ of $J$ in $Z$ such that

$$
G(x)=\lim _{n \rightarrow \infty} \frac{1}{\eta_{i}^{27 n}} f\left(\eta_{i}^{n} x\right)
$$

for all $x \in X$. In order to show that $G$ satisfies (11), replace $(x, y)$ by $\left(\eta_{i}^{n} x, \eta_{i}^{n} y\right)$ in (63) and divide by $\eta_{i}^{27 n}$, we get

$$
\begin{aligned}
\left\|G_{27}(x, y)\right\| & =\lim _{n \rightarrow \infty} \frac{1}{\eta_{i}^{27 n}}\left\|D f_{27}\left(\eta_{i}^{n} x, \eta_{i}^{n} y\right)\right\| \\
& \leq \lim _{n \rightarrow \infty} \frac{1}{\eta_{i}^{27 n}} \gamma\left(\eta_{i}^{n} x, \eta_{i}^{n} y\right) \\
& =0
\end{aligned}
$$

for all $x, y \in X$ and so the mapping $G$ is Pinelas-Septoicosic. By property (B3), $G$ is the unique fixed point of $J$ in the set

$$
\Delta=\{G \in Z: d(f, G)<\infty\}
$$

Finally, by property (B4), we arrive

$$
\|f(x)-G(x)\| \leq \frac{L^{1-i}}{1-L}
$$

Hence the proof is completed.

Using Theorem 5, we prove the following corollary concerning the stability of (11).

Remark 2. Let $D f_{27}: X \rightarrow Y$ be a mapping. If there exist real numbers $\psi$ and $\lambda$ such that

$$
\left\|D f_{27}(x, y)\right\| \leq \begin{cases}\psi, & \\ \psi\left\{\|x\|^{\lambda}+\|y\|^{\lambda}\right\}, & \lambda \neq 27 \\ \psi\|x\|^{\lambda}\|y\|^{\lambda} & \lambda \neq \frac{27}{2} \\ \psi\left\{\|x\|^{\lambda}\|y\|^{\lambda}+\left\{\|x\|^{2 \lambda}+\|y\|^{2 \lambda}\right\}\right\}, & \lambda \neq \frac{27}{2}\end{cases}
$$


for all $x, y \in X$, then there exists a unique Pinelas-Septoicosic mapping $G: X \rightarrow Y$ such that

$$
\|f(x)-G(x)\| \leq\left\{\begin{array}{l}
\frac{\psi_{C}}{\left|2^{27}-1\right|}, \\
\frac{\psi_{S}}{\left|2^{27}-2^{\lambda}\right|} \\
\frac{\psi_{P}}{\left|2^{27}-2^{2 \lambda}\right|} \\
\frac{\psi_{S P}}{\left|2^{27}-2^{2 \lambda}\right|}
\end{array}\right.
$$

where, $\psi_{C}=\frac{134217729 \psi}{1.088886945 \times 10^{28}}$

$$
\begin{aligned}
\psi_{S}= & \frac{\psi\|x\|^{\lambda}}{1.088886945 \times 10^{28} .2^{\lambda}}\left[14^{\lambda}+27.13^{\lambda}+352.12^{\lambda}+2952.11^{\lambda}+17901.10^{\lambda}\right. \\
& +83655.9^{\lambda}+313560.8^{\lambda}+968760.7^{\lambda}+2516085.6^{\lambda}+5574855.5^{\lambda}+10656360.4^{\lambda} \\
& \left.+17724720.3^{\lambda}+25820146.2^{\lambda}+167313923\right], \\
\psi_{P}= & \frac{\psi\|x\|^{2 \lambda}}{1.088886945 \times 10^{28} \cdot 2^{2 \lambda}}\left[14^{\lambda}+27.13^{\lambda}+352.12^{\lambda}+2952.11^{\lambda}+17901.10^{\lambda}\right. \\
& +83655.9^{\lambda}+313560.8^{\lambda}+968760.7^{\lambda}+2516085.6^{\lambda}+5574855.5^{\lambda} \\
& \left.+10656360.4^{\lambda}+17724720.3^{\lambda}+25820146.2^{\lambda}+33096195\right], \\
\psi_{S P}=\quad & \quad \psi\|x\|^{2 \lambda} \\
& \\
+ & 8388855.9^{2 \lambda}+313560.8^{2 \lambda}+968760.7^{2 \lambda}+2516085.6^{2 \lambda}+5574855.5^{2 \lambda}+10656360.4^{2 \lambda} \\
+ & \left.17724720.3^{2 \lambda}+25820146.2^{2 \lambda}+167313923\right]+\left[14^{\lambda}+27.13^{\lambda}+352.12^{\lambda}+2952.11^{\lambda}\right. \\
+ & 17901.10^{\lambda}+83655.9^{\lambda}+313560.8^{\lambda}+968760.7^{\lambda}+2516085.6^{\lambda}+5574855.5^{\lambda} \\
+ & \left.\left.10656360.4^{\lambda}+17724720.3^{\lambda}+25820146.2^{\lambda}+33096195\right]\right\}
\end{aligned}
$$

for all $x \in X$.

Proof. Let

$$
\gamma(x, y)=\left\{\begin{array}{l}
\psi \\
\psi\left\{\|x\|^{\lambda}+\|y\|^{\lambda}\right\} \\
\psi\|x\|^{\lambda}\|y\|^{\lambda} \\
\psi\left\{\|x\|^{\lambda}\|y\|^{\lambda}+\left\{\|x\|^{2 \lambda}+\|y\|^{2 \lambda}\right\}\right\}
\end{array}\right.
$$

for all $x, y \in X$. Now

$$
\begin{aligned}
& \frac{1}{\eta_{i}^{27 n}} \gamma\left(\eta_{i}^{n} x, \eta_{i}^{n} y\right)=\left\{\begin{array}{l}
\frac{\psi}{\eta_{i}^{27 n}} \\
\frac{\psi}{\eta_{i}^{27 n}}\left\{\left\|\eta_{i}^{n} x\right\|^{\lambda}+\left\|\eta_{i}^{n} y\right\|^{\lambda}\right\} \\
\frac{\psi}{\eta_{i}^{27 n}}\left\|\eta_{i}^{n} x\right\|^{\lambda}\left\|\eta_{i}^{n} y\right\|^{\lambda} \\
\frac{\psi}{\eta_{i}^{27 n}}\left\{\left\|\eta_{i}^{n} x\right\|^{\lambda}\left\|\eta_{i}^{n} y\right\|^{\lambda}+\left\{\left\|\eta_{i}^{n} x\right\|^{2 \lambda}+\left\|\eta_{i}^{n} y\right\|^{2 \lambda}\right\}\right\}
\end{array}\right. \\
& =\left\{\begin{array}{l}
\rightarrow 0 \text { as } n \rightarrow \infty \\
\rightarrow 0 \text { as } n \rightarrow \infty \\
\rightarrow 0 \text { as } n \rightarrow \infty \\
\rightarrow 0 \text { as } n \rightarrow \infty
\end{array}\right.
\end{aligned}
$$

Thus, (62) holds. On the other hand

$$
D(x, x)=\alpha\left(\frac{x}{2}, \frac{x}{2}\right)
$$


for all $x \in X$. It follows from (70), we get

$$
D(x, x)=\alpha\left(\frac{x}{2}, \frac{x}{2}\right)=\left\{\begin{array}{l}
\psi_{C} \\
\psi_{S} \\
\psi_{P} \\
\psi_{S P}
\end{array}\right.
$$

for all $x \in X$. Similarly by (70), we prove

$$
\frac{1}{\eta_{i}^{27}} D\left(\eta_{i} x, \eta_{i} x\right)=\left\{\begin{array}{l}
\eta_{i}^{-27} \psi_{C} \\
\eta_{i}^{\lambda-27} \psi_{S} \\
\eta_{i}^{2 \lambda-27} \psi_{P} \\
\eta_{i}^{2 \lambda-27} \psi_{S P}
\end{array}\right.
$$

Hence, the inequality (65) holds for

i) $L=\eta_{i}^{-27}$ if $i=0$ and $L=\eta_{i}^{27}$ if $i=1$;

ii) $L=\eta_{i}^{\lambda-27}$ for $p<27$ if $i=0$ and $L=\eta_{i}^{27-\lambda}$ for $p>27$ if $i=1$;

iii) $L=\eta_{i}^{2 \lambda-27}$ for $2 \lambda<27$ if $i=0$ and $L=\eta_{i}^{2 \lambda-27}$ for $2 \lambda>27$ if $i=1$;

iv) $L=\eta_{i}^{2 \lambda-27}$ for $2 \lambda<27$ if $i=0$ and $L=\eta_{i}^{2 \lambda-27}$ for $2 \lambda>27$ if $i=1$.

Now, from (65), we show the following cases for condition (i).

i) $L=\eta_{i}^{-27}$ if $i=0$

$$
\|f(x)-G(x)\| \leq\left(\frac{L^{1-i}}{1-L}\right) D(x, x)=\left(\frac{2^{-27}}{1-2^{-27}}\right) \psi_{C}=\frac{\psi_{C}}{2^{27}-1}
$$

and for $L=\eta_{i}^{27}$ if $i=1$

$$
\|f(x)-G(x)\| \leq\left(\frac{L^{1-i}}{1-L}\right) D(x, x)=\left(\frac{1}{1-2^{27}}\right) \psi_{C}=\frac{\psi_{C}}{1-2^{27}}
$$

ii) $L=\eta_{i}^{\lambda-27}$ for $p<27$ if $i=0$

$$
\|f(x)-G(x)\| \leq\left(\frac{L^{1-i}}{1-L}\right) D(x, x)=\left(\frac{2^{\lambda-27}}{1-2^{\lambda-27}}\right) \psi_{S}=\frac{2^{\lambda} \cdot \psi_{S}}{2^{27}-2^{\lambda}}
$$

and for $L=\eta_{i}^{27-\lambda}$ for $p>27$ if $i=1$

$$
\|f(x)-G(x)\| \leq\left(\frac{L^{1-i}}{1-L}\right) D(x, x)=\left(\frac{1}{1-2^{27-\lambda}}\right) \psi_{S}=\frac{2^{\lambda} \cdot \psi_{S}}{2^{\lambda}-2^{27}}
$$

iii) $L=\eta_{i}^{2 \lambda-27}$ for $2 \lambda<27$ if $i=0$

$$
\|f(x)-G(x)\| \leq\left(\frac{L^{1-i}}{1-L}\right) D(x, x)=\left(\frac{2^{2 \lambda-27}}{1-2^{2 \lambda-27}}\right) \psi_{P}=\frac{2^{2 \lambda} \cdot \psi_{P}}{2^{27}-2^{2 \lambda}}
$$

and for $L=\eta_{i}^{2 \lambda-27}$ for $2 \lambda>27$ if $i=1$

$$
\|f(x)-G(x)\| \leq\left(\frac{L^{1-i}}{1-L}\right) D(x, x)=\left(\frac{1}{1-2^{27-2 \lambda}}\right) \psi_{P}=\frac{2^{2 \lambda} \cdot \psi_{P}}{2^{2 \lambda}-2^{27}}
$$

Finally, the proof of (65) for condition (iv) is similar to the condition (ii) and (iii).

\section{Conclusion}

We derived the general solution for the Septoicosic functional equation and investigated its Hyers-Ulam stability results in Banach spaces using two different methods of direct method and fixed point method. Author

Contributions: All authors contributed equally to the writing of this paper. All authors read and approved the final manuscript.

Conflicts of Interest: "The authors declare no conflict of interest." 


\section{References}

[1] Gavruta, P. (1994). A generalization of the Hyers-Ulam-Rassias stability of approximately additive mappings. Journal of Mathematical Analysis and Applications, 184(3), 431-436. https:/ / doi.org/10.1006/jmaa.1994.1211

[2] Kim, S. H. (2016). On the stability of a nonic functional equation in quasi-normed spaces. Journal of Advances in Physics, 12(3), 4368-4374. https:/ / doi.org/10.24297/jap.v12i3.47

[3] Hyers, D. H. (1941). On the stability of the linear functional equation. Proceedings of the National Academy of Sciences of the United States of America, 27(4), 222-224.

[4] Ulam, S. M. (1940). Problems in Modern Mathematics, Chapter VI. Science Ed., Wiley, New York.

[5] Aoki, T. (1950). On the stability of the linear transformation in Banach spaces. Journal of the Mathematical Society of Japan, 2(1-2), 64-66. https:/ / doi.org/10.2969/jmsj/00210064

[6] Rassias, T. M. (1978). On the stability of the linear mapping in Banach spaces. Proceedings of the American Mathematical Society, 72(2), 297-300. https://doi.org/10.1090/S0002-9939-1978-0507327-1

[7] Govindan, V., Murthy, S., \& Saravanan, M. (2017). Generalized Ulam-Hyers stability of two types of n-dimensional quadratic functional equation in Banach space: direct and fixed point methods. Int. J. Math. Trends and Its Tech., 51, 396-403.

[8] Govindan, V., Balasubramanian, G., \& Muthamilarasi, C. (2018). Generalized stability of cubic functional equation, Inter. J. Scientific Eng. Research, 2, 74-82.

[9] Govindan, V., Murthy, S., \& Kokila, G. (2018). Hyers-Ulam Stability of AQ Functional Equation. International Journal Of Mathematics And Computer Research, 6(01), 1852-1859. https:/ / doi.org/10.31142/ijmcr.v6i01.2

[10] Govindan, V., \& Tamilvanan, K. (2018). Stability of functional equation in banach space: using two different methods. Int. J. Math. Appl., 6(1-C), 527-536.

[11] Murthy, S., \& Govindhan, V. (2017). General solution and generalized HU (Hyers - Ulam) statbility of new dimension cubic functional equation in fuzzy ternary banach algebras: using two different methods. Int. J. Pure and Appl. Math., 113.

[12] Murthy, S., Govindan, V., \& Saravanan, M. (2018). Generalized Hyers-Ulam stability of mixed type functional equation in banach and fuzzy banach space using direct and fixed point methods. Int. J. Scientific Eng. Research, 1,01-11.

[13] Ravi, K., Rassias, J. M., Sandra Pinelas \& Jamuna, R. (2014). A fixed point approach to the stability equation in paranormed spaces. Pan American Mathematical J., 24, 61-84.

[14] Xu, T. Z., \& Rassias, J. M. (2013). Approximate septic and octic mappings in quasi- $\beta$-normed spaces. J. Comp. Anal. Appl, 15(6), 1110-1119.

[15] Czerwik, S. (1992). On the stability of the quadratic mappings in normed spaces. Abh. Math. Sem. Univ Hamburg, 62, 59-64. https://doi.org/10.1007/BF02941618

[16] Jun, K. W., \& Kim, H. M. (2002). The generalized Hyers-Ulam-Rassias stability of a cubic functional equation. Journal of Mathematical Analysis and Applications, 274(2), 867-878. https://doi.org/10.1016/S0022-247X(02)00415-8

[17] Lee, S. H., Im, S. M., \& Hwang, I. S. (2005). Quartic functional equations. Journal of Mathematical Analysis and Applications, 307(2), 387-394. https:// doi.org/10.1016/j.jmaa.2004.12.062

[18] Ulam, S. M. (1960). A collection of mathematical problems (Vol. 8). Interscience Publishers. New York.

[19] XXu, T. Z., Rassias, M. J., \& Xin Xu, W. (2012). A fixed point approach to the intuitionistic fuzzy stability of quintic and sextic functional equations. Iranian Journal of Fuzzy Systems, 9(5), 21-40. https://doi.org/10.22111/IJFS.2012.102

[20] Rassias, J. M., \& Eslamian, M. (2015). Fixed points and stability of nonic functional equation in quasi- $\beta$-normed spaces. Cont. Anal. Appl. Math, 3(2), 293-309. https://doi.org/10.18532/caam.38853

[21] Bodaghi, A., Park, C., \& Rassias, J. M. (2016). Fundamental stabilities of the nonic functional equation in intuitionistic fuzzy Normed spaces. Commun. Korean Math. Soc, 31(4), 729-743. http://dx.doi.org/10.4134/CKMS.c150147

[22] Rassias, J. M., Arunkumar, M., Sathya, E., \& Namachivayam, T. (2016). Various generalized Ulam-Hyers stabilities of a nonic functional equations. Tbilisi Mathematical Journal, 9(1), 159-196. https://doi.org/10.1515/tmj-2016-0008

[23] Ravi, K., Rassias, J. M., \& Kumar, B. S. (2016). Ulam-Hyers stability of undecic functional equation in quasi- $\beta$-normed spaces: Fixed point method. Tbilisi Mathematical Journal, 9(2), 83-103. https://doi.org/10.1515/tmj-2016-0022

[24] Murali, R., \& Antony Raj, A. (2017). Stability of duodecic functional equations in multi-banch spaces.Int. J. Computing Algorithm, 6(1), 31-32.

[25] Pinelas, S., RAMDOSS, M., \& Veeramani, V. (2017). Stability of tredecic functional equation in matrix normed spaces. Journal of Advances in Mathematics, 13(2), 7135-7146. https://doi.org/10.24297/jam.v13i2.5947

[26] Ramdoss, M., Park, C., Veeramani, V., \& Cho, Y. (2019). A septendecic functional equation in matrix normed spaces. J. Computational Analysis and Appl., 26 (2).

[27] Nazarianpoor, M., Rassias, J. M., \& Sadeghi, G. (2018). Stability and nonstability of octadecic functional equation in multi-normed spaces. Arabian Journal of Mathematics, 219-228. https:/ /doi.org/10.1007/s40065-017-0186-0 
[28] Murali, R., \& Antony Raj, A. (2017). Ulam-Hyers stability of nonadecic functional equation in multi-banach spaces. Int. J. Pure and Appl. Math., 117 (12), 63-72.

[29] Murali, R., Sandra Pinelas \& Antony Raj, A. (2017). General solution and a fixed point approach to the Ulam Hyers stability of viginti duo functional equation in multi-banach spaces.IOSR J. Math.,, 13 (4-II), 48-59.

[30] Rassias, J. M., Murali, R.,Rassias, M. J., \& Antony, R.. A. (2017). General solution, stability and non-stability of quattuorvigintic functional equation in multi-banach spaces. Inter. J. Math. Appl., 5(2-A), 181-194.

[31] Bodaghi, A., Park, C., \& Rassias, J. M. (2016). Fundamental stabilities of the nonic functional equation in intuitionistic fuzzy normed spaces. Commun. Korean Math. Soc, 31(4), 729-743. http:/ /dx.doi.org/10.4134/CKMS.c150147

[32] Govindan, V. (2018). Stability solution of quadratic functional equation in Banach space using direct method. Inter. J. Scientific Eng. Research, 1, 01-06.

[33] Govindan, V., Murthy, S.,\& Kokila, G. (2018). Fixed point and stability of icosic functional equation in quasi beta normed spaces. Malaya J. Mathematic, 6 , 261-275. http:/ /dx.doi.org/10.26637/MJM0601/0030

[34] Govindan, V., Murthy, S.,\& Saravanan, M. (2018). Solution and stability of a cubic type functional equation: using direct and fixed point methods. Kragujevac J. Math, 44(1), 7-26.

[35] Murali, R., Sandra Pinelas \& Vithya, V. (2017). The stability of viginti unus functional equation in various spaces. Global J. Pure and Appl. Math., 13, 5735-5759.

[36] Murthy, S., Govindhan, V., \& Sree Shanmuga Velan, M. (2016). Solution and stability of two types of n-dimensional quartic functional equation in generalized 2-normed spaces. Int. J. Pure and Applied Math., 111, 249-272.

[37] Murthy, S., Govindhan, V., \& Sree Shanmuga Velan, M. (2017). Generalized U - H stability of new n - type of additive quartic functional equation in non - archimedean space. Int. J. Math. Appl., 5 ( 2-A), 1- 11.

[38] Pinelas, S., Narasimman, P., \& Ravi, K. (2015). Stability of Pythagorean mean functional equation. Global Journal of Mathematics, $1(4$ (2015)), 398-411.

[39] Rassias, J. M. (1982). On approximately of linear mappings.J. Functional Anal., USA, 46, 126-130.

[40] Rassias, J. M. (2000). Solution of the Ulam problem for cubic mappings. Analele Universitatii din Timisoara. Seria Matematica-Informatica, 38(1), 121-132.

[41] Ravi, K., Rassias, J. M., Pinelas, S., Militar, A., \& Narasimman, P. (2014). The Stability of a Generalized Radical Reciprocal Quadratic Functional Equation in Felbin's Space. PanAmerican Mathematical Journal, 24(1), 75-92.

[42] Sandra Pinelas, Govindan, V., \& Tamilvanan, K. (2018). Stability of non- additive functional equation. IOSR J. Math., 14( 2-I), 60-78.

[43] Pinelas, S., Govindan, V., \& Tamilvanan, K. (2018). Pfister 16-Square Quadratic Functional Equation. Global Journal of Mathematics, 12(1), 760-772.

[44] Pinelas, S., Govindan, V., \& Tamilvanan, K. (2018). Stability of a quartic functional equation. Journal of Fixed Point Theory and Applications, 20(4), 148. http:/ / dx.doi.org/10.1007/s11784-018-0629-z.

[45] Xu, T., Rassias, J., Rassias, M., \& Xu, W. (2011). A fixed point approach to the stability of quintic and sextic functional equations in quasi- $\beta$-normed spaces. Journal of Inequalities and Applications, $2010(1), 423231$. https:/ / doi.org/10.1155/2010/423231

[46] Baker, J. (2005). A general functional equation and its stability. Proceedings of the American Mathematical Society, 133(6), 1657-1664. https:/ /doi.org/10.1090/S0002-9939-05-07841-X 